\title{
Atom Clusters in Decagonal Quasicrystals: Local Structures and Long-range Arrangements Revealed by High-resolution Electron Microscopy
}

\author{
Wei Sun and Kenji Hiraga \\ Institute for Materials Research, Tohoku University, Katahira 2-1-1, Aoba-ku Sendai 980-8577, Japan
}

High-resolution electron microscopy (HREM) has been played an important role in structural study of decagonal quasicrystals with different periods. On the other hand, the high-angle annular detector dark-field (HAADF) method is another powerful imaging technique to obtain structural information in atom scale. In order to reveal intrinsic structural features of decagonal quasicrystals and to understand their formation process, structural information of both basic structural units (nano-sized atom clusters) and their arrangements in a wider region are quite essential. In the present study, long-range arrangement of atom clusters in a wide region (larger than $100 \times 100 \mathrm{~nm}^{2}$ ) for a highly ordered Al-Ni-Ru D-phase with $1.6 \mathrm{~nm}$ periodicity has been revealed and compared with that for an Al-Pd-Mn D-phase with $1.2 \mathrm{~nm}$ periodicity on the basis of HREM. In addition, the local arrangement of TM atoms in the Al-Ni-Ru D-phase, revealed by HAADF observations, is also presented.

Alloy ingots with nominal compositions of $\mathrm{Al}_{75} \mathrm{Ni}_{15} \mathrm{Ru}_{10}$ and $\mathrm{Al}_{70} \mathrm{Pd}_{13} \mathrm{Mn}_{17}$ were prepared by arc-melting high purity metals under an argon atmosphere. Parts of the ingots obtained were sealed in evacuated quartz tubes and various heat treatments were made in order to form Dphases. The specimens for transmission electron microscope observations were prepared by dispersing crushed ingot fragments on the microgrids covered by holey carbon films. HREM observations were performed using a $400 \mathrm{kV}$ electron microscope (JEM-4000EX) with a point resolution of $0.17 \mathrm{~nm}$. HAADF observations were carried out using a JEM-2010F electron microscope equipped with a HAADF detector.

Fig. 1 shows the typical tenfold symmetry electron diffraction patterns taken from an Al-Ni-Ru D-phase with $1.6 \mathrm{~nm}$ periodicity and from an Al-Pd-Mn D-phase with $1.2 \mathrm{~nm}$ periodicity, respectively. In contrast to Fig. 1(b) of the Al-Pd-Mn D-phase which contains apparent linear phason strain, Fig. 1(a) of the Al-Ni-Ru D-phase shows a large number of sharp diffraction spots located at correct tenfold symmetry positions. This indicates that Al-Ni-Ru D-phase is a highly ordered decagonal quasicrystal. Fig. 2(a) shows a typical HREM image of the Al-Ni-Ru D-phase, taken along the tenfold symmetry axis. In the image, characteristic bright ring contrasts corresponding to atom clusters are clearly seen. They are arranged uniformly, but aperiodically with $2 \mathrm{~nm}$ distance between them to form a pentagonal tiling as outlined with white lines in the observed image. The local TM atom arrangement in the tiling has been clearly revealed by HAADF observation as seen in Fig. 2(b) (in which bright dot contrasts correspond to TM atoms). A part of tiling formed by connecting the centers of atom clusters is also outlined with white lines in Fig. 2(b). HREM examinations in wide sample regions show that the long-range arrangement characteristic of atom clusters in the Al-Ni-Ru D-phase is completely different from that in the Al-Pd-Mn D-phase. On the basis of the perpendicular space analysis of the tiling structure observed in a wide region, we conclude that long-range arrangement of atom clusters in the Al-Ni-Ru D-phase with $1.6 \mathrm{~nm}$ periodicity can form a Penrose-tiling-like quasiperiodic structure which is almost free of linear phase strain. 

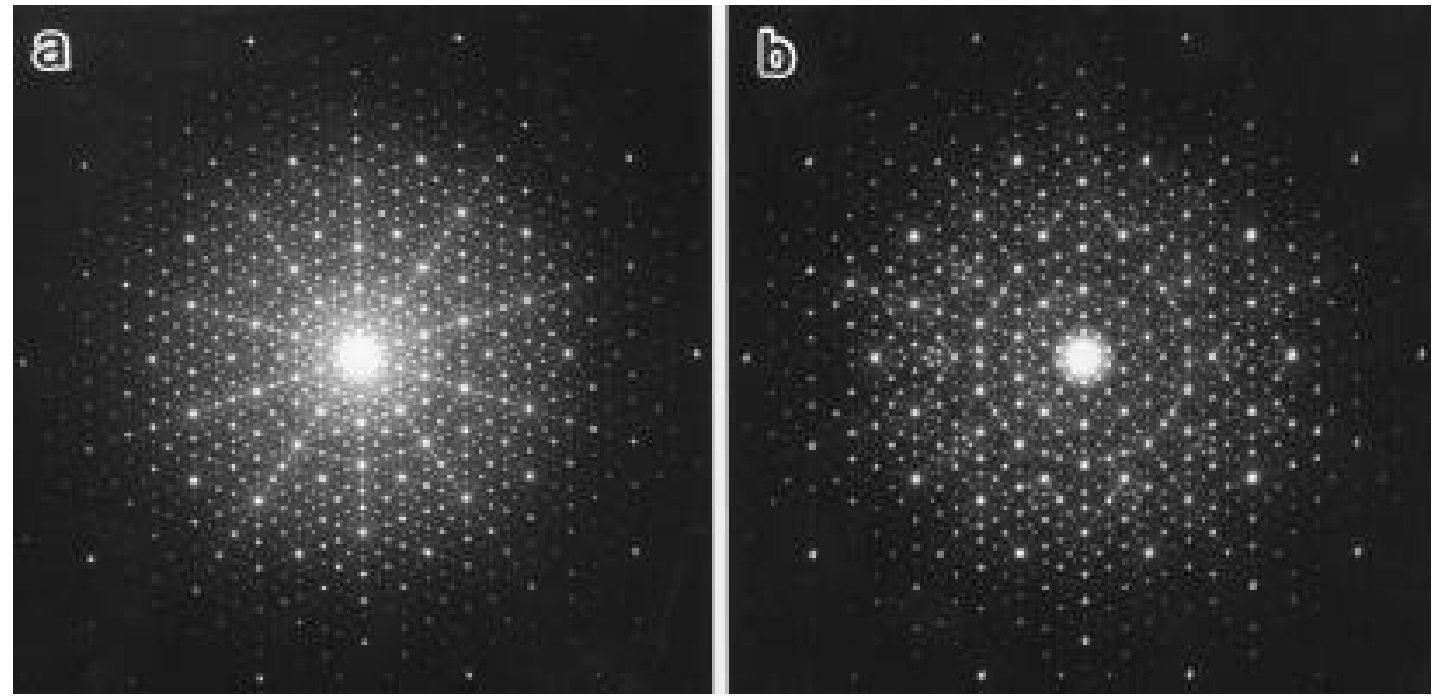

FIG. 1 Typical electron diffraction patterns taken from an Al-Ni-Ru D-phase with $1.6 \mathrm{~nm}$ periodicity (a) and from an Al-Pd-Mn D-phase with $1.2 \mathrm{~nm}$ periodicity (b). The incident beam is parallel to their tenfold axes.

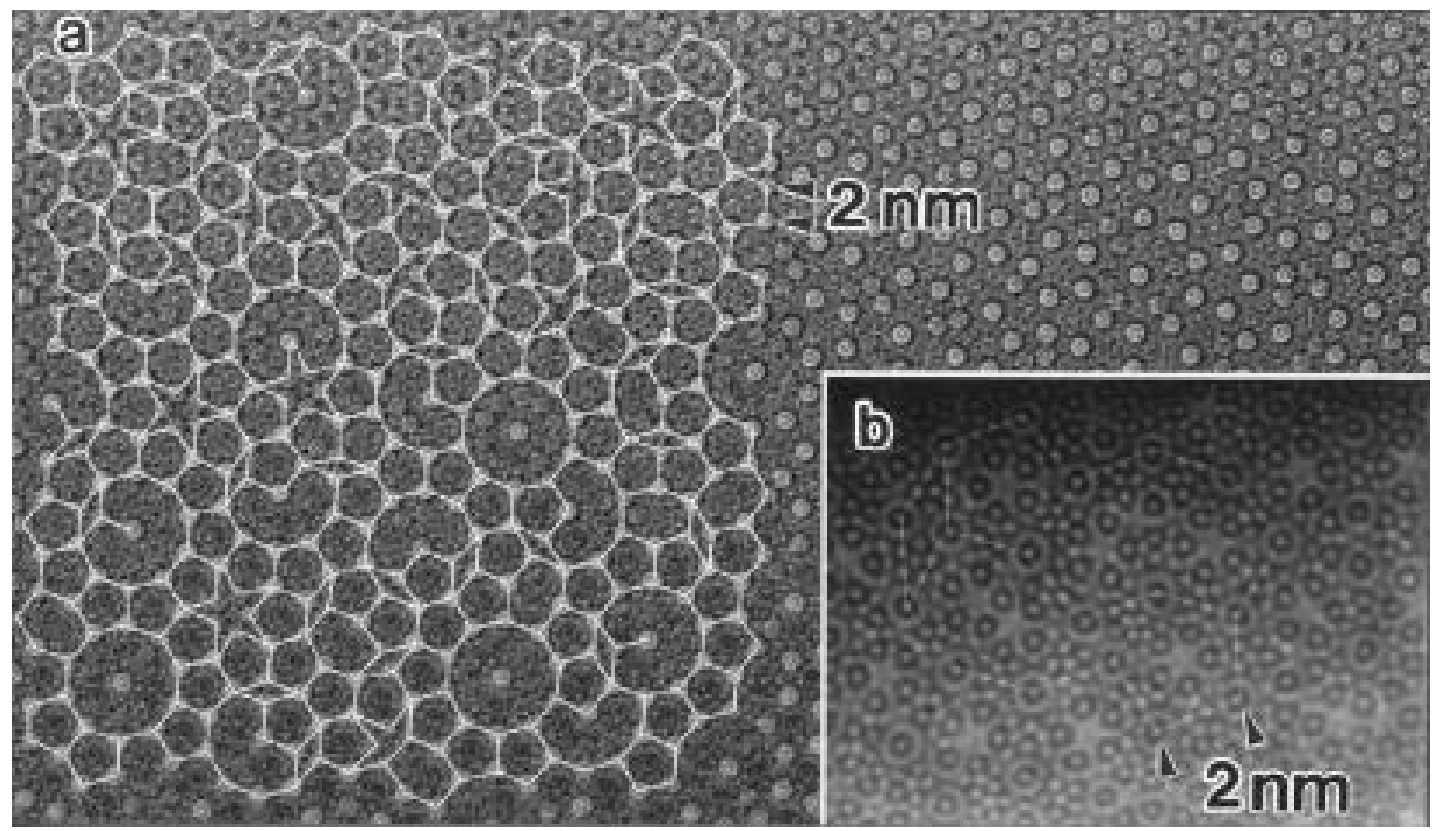

FIG. 2 HREM (a) and HAADF (b) images of the Al-Ni-Ru decagonal quasicrystal with 1.6 $\mathrm{nm}$ periodicity formed in the $\mathrm{Al}_{75} \mathrm{Ni}_{15} \mathrm{Ru}_{10}$ alloy annealed at $890^{\circ} \mathrm{C}$ for $24 \mathrm{~h}$. Both were taken with the incident beam parallel to the tenfold axis. A part of tiling structure formed by the arrangement of atom clusters is outlined in both (a) and (b). 